\title{
Sleep and the Family Doctor: Time to Lead
}

Adam J. Sorscher, MD

In this issue of the Fournal of the American Board of Family Medicine, the American Board of Family Medicine presents 4 original research articles about sleep-related topics. This signals a growing recognition of the role of sleep disorders/complaints in health and well-being and of sleep medicine's relevance to the practice of family medicine.

There are several themes that arise in these research reports. The first is the staggering prevalence of sleep disorders/complaints in the primary care setting. This is succinctly stated by Mold et $\mathrm{al}^{1}$ : "nearly all patients who visit [primary care clinics] regularly have sleep-related symptoms." Pigeon et $\mathrm{al}^{2}$ are in the same ballpark statistically when, using a validated instrument, they find a $71 \%$ prevalence of disturbed sleep among urban patients. Though they studied a healthier population presenting for preventive medicine, Grover et $\mathrm{al}^{3}$ nevertheless discover that more than $50 \%$ of patients either reported a sleep complaint in a review of systems questionnaire or were found to be at high risk for sleep apnea judging by responses to the Berlin Questionnaire.

A second theme is the impact of sleep disorders. The negative consequences of disturbed sleep involve multiple areas of health, including the cardiovascular, metabolic, hormonal, and immune systems; mental health; and cognitive function. Tandeter et al's ${ }^{4}$ study, simply by virtue of its topic, reveals the protean side of sleep disorders: they frequently masquerade as other diagnoses, in this case as benign prostatic enlargement. Similarly, previous reports have indicated that primary sleep disorders may be incorrectly diagnosed as depression, chronic headache, or attention deficit disorder

From the Department of Community and Family Medicine, Dartmouth Medical School, Hanover, NH.

Funding: none.

Conflict of interest: none declared.

Corresponding author: Adam J. Sorscher, MD, Department of Community and Family Medicine, Dartmouth Medical School, One Medical Center Drive, Lebanon, NH 93756 (E-mail: adam.sorscher@dartmouth.edu). because of an overlap in symptoms. These 2 considerations - burden of sleep disorders to multiple physiologic systems and the potential for misdiagnosis-compel us to improve our recognition and response to sleep disorders/complaints.

The third theme is the apparent reticence on the part of family physicians to take action when confronted with sleep complaints. Perhaps they are overwhelmed by the sheer volume of the complaints. Grover et $\mathrm{al}^{3}$ report that only $11 \%$ of individuals who reported sleep complaints underwent any subsequent diagnostic testing. This study includes physicians who regard themselves as educated about and "on board" with the significance of obstructive sleep apnea, and the authors conclude that there is a gap between "factual knowledge and appropriate clinical behaviors."

\section{Why is It So Difficult to Take Action When Confronted With Sleep Disorders/Complaints?}

At present, there are important barriers to providing effective care for sleep disorders. For example, we know that insomnia is the most prevalent sleep disorder and that the most effective treatment for insomnia is cognitive-behavioral therapy (CBT-I). Yet there is a woeful lack of trained providers of CBT-I, and many large metropolitan areas may not have even a single highly-trained CBT-I specialist. Though sleep apnea may lend itself well to screening and referral protocols for diagnosis, there are many obstacles to effective treatment: long wait times for testing, the inability of many people to afford the high cost of sleep studies, frequent frustration with the standard therapy (continuous positive airway pressure), and dissatisfaction with medical equipment companies and sleep specialists' care of patients after the diagnostic test has been performed (and billed for).

Sleep complaints by nature often are a troublesome area for family physicians. Most of our readers are likely to have experienced the dissonant moment when, after responding to a long list of patient complaints and preparing to exit the exam- 
ination room, the patient adds on one last request: Can you give me something to help me sleep? The problem calls for a willingness to follow up with many additional and likely time-consuming questions, time that is often unavailable. Most of the sleep complaints are like fatigue or dizziness - conditions for which there is a long list of possible causes and contributing factors.

This is further complicated by the primary treatment for sleep disorders requested by patients: sleeping pills. The medicines available are sometimes addictive, may be associated with negative outcomes (such as falling), and are generally not recommended for long-term use. Thus, there is no "easy fix" in just writing a prescription.

Basically, many if not most sleep difficulties result from the American lifestyle, something the individual physician is unlikely to cure soon. Voluntary sleep restriction is very prevalent in our overworked, overstimulated culture. On average, American adults obtained 9.1 hours of sleep before the advent of the electric light, but recent analyses indicate that the current average is less than 7 hours. The trend toward shortchanging sleep in favor of other activities has important implications for human health, including quality of life, performance, and even mortality rates. Therefore, we should place proper sleep behavior on par with other basic elements of a healthy lifestyle, such as nutritious eating, physical activity, and fostering good mental health. All of these concerns principally are in the domain of the patient, with some help and support from family physicians.

In light of such obstacles to addressing sleep disorders/complaints and to bridge the gap between knowledge and action on behalf of the patient, the question is, what is necessary to translate increasing knowledge about the harms of disturbed sleep into more effective patient care? Mold et $\mathrm{al}^{1}$ call for more "...published guidelines...that provide specific recommendations about whether and how to screen." Another piece of the solution is to improve the office tools used to screen, document, and act on sleep complaints. Efficiency is crucial in the crucible of the office encounter, where minutes count. Mold et $\mathrm{al}^{1}$ and Grover et $\mathrm{al}^{3}$ both indicate that review of systems questionnaires need to include a more thorough set of inquiries about sleep symptoms.

Though these are steps in the right direction, even thorough, diligent screening and documenta- tion does not always lead to appropriate action on behalf of the patient. A fundamental shift in attitude is also necessary; institutionally, family medicine must seek a greater level of engagement in the field of sleep medicine. Family doctors are overwhelmingly the first responders to sleep complaints, be it sleepless new parents, teenagers who cannot be roused for school, middle-aged snorers with hypersomnolence, or postmenopausal women experiencing insomnia.

Family medicine is at a juncture where we increasingly acknowledge the relevance of sleep disorders to health and well-being but are struggling to figure out how best to manage them. In the future, an important barometer of family medicine's leadership in sleep medicine will be the number of physicians from our ranks who pursue the certificate of added qualifications in sleep medicine and thereby participate as experts in the field. There are very few family medicine board-certified physicians thus far who have pursued specialty training in sleep medicine. At present there is a total of 60 physicians who are dual-boardcertified in family medicine and sleep medicine; this constitutes less than $2 \%$ of the total number of sleep medicine specialists. The field is currently dominated by pulmonologists (approximately 67\% of sleep specialists) even though obstructive sleep apnea is just a small piece of the sleep/health puzzle compared with insomnias and voluntary insufficient sleep.

Family medicine should stake a greater claim to the field of clinical sleep medicine. We should encourage more family medicine physicians to pursue the certificate of added qualification in sleep medicine and increase our residents' exposure to and training for sleep disorders. One chief driving force behind these changes likely is the continuing accumulation of evidence that successful treatment of sleep disorders will improve patient care, lower morbidity and mortality, and save health care dollars. That is why this issue of Fournal of the American Board of Family Medicine, featuring these reports of original sleep medicine research, is so exciting!

\section{References}

1. Mold JW, Quattlebaum C, Schinnerer E, Boeckman L, Orr W, and Hollabaugh K. Identification by primary care clinicians of patients with obstructive sleep apnea: a practice-based research (PBRN) study. J Am Board Fam Med 2011;24:138-145. 
2. Pigeon WR, Heffner K, Duberstein P, Fiscella K, Moynihan J, Chapman BP. Elevated sleep disturbance among blacks in an urban family medicine practice. J Am Board Fam Med 2011;24:161-168.

3. Grover ML, Mookadam M, Armas D, et al. Identifying patients at risk for obstructive sleep apnea in a primary care practice. J Am Board Fam Med 2011; 24:152-160.

4. Tandeter H, Gendler S, Dreiher J, Tarasiuk A. Nocturic episodes in patients with benign prostatic enlargement may suggest the presence of obstructive sleep apnea. J Am Board Fam Med 2011;24:146-151. 